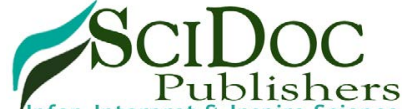

International Journal of Clinical Dermatology \& Research (IJCDR)

ISSN 2332-2977

\title{
Effect of 'Treating Winter Disease in Summer' Prescription on Skin Immune Barrier and Systemic Immunity in a Guinea Pig Model of Asthma
}

Research Article

Shijian Xiang, ${ }^{1, \#}$, Huoji Chen ${ }^{1, \#}$, Ting Zuo ${ }^{1}$, Junhui Hong ${ }^{1}$, Siwei Cao ${ }^{1}$, Shifa Ruan ${ }^{1}$, Lidong Weng ${ }^{1}$, Hongxia Zhu ${ }^{2, *}$, Qiang Liu ${ }^{1, *}$

${ }^{1}$ School of Traditional Chinese Medicine, Southern Medical University, Guangzhou, PR China.

${ }^{2}$ Combining Traditional Chinese and Western Medicine Hospital, Southern Medical University, Guangzhou, PR China.

\# These Authors Contributed Equally to this Work

\section{Abstract}

Objective: To investigate the effect of transdermal and topical application of 'Treating Winter Disease in Summer' prescription on skin immune barrier and systemic immunity of asthma guinea-pigs, and its underlying mechanism.

Methods: Thirty guinea-pigs were randomized into blank control group $(n=10)$, OVA sensitization model group $(n=10)$ and transdermal drug delivery (TDD) patch therapeutic group $(n=10)$. TDD patch therapeutic group animals were received treatment by 'Treating Winter Disease in Summer' prescription for transdermal and topical application. Lung tissue of guinea-pigs was being H\&E stained. The expression of IL-10, IFN- $\alpha$ and IFN- $\gamma$ in the skin and in the serum of guinea-pigs was examined, respectively.

Results: H\&E staining of lung tissue showed that the model of asthma in guinea-pig was successfully established, and it also showed that airway condition of asthma animals was significantly improved in OVA-sensitized animals treated with TDD patches; Results also revealed that the expression levels of immune factors such as IL-10, IFN- $\alpha$ and IFN- $\gamma$ were markedly increased following TDD patches treatments.

Conclusion: This study demonstrates that TDD patches reduces airway hyperresponsiveness and lung inflammation in OVAsensitized animals, a finding consistent with a increase in IL-10, IFN- $\alpha$ and IFN- $\gamma$ expression levels in serum and in the skin. The present data suggest that transdermal delivery of TDD patches improve skin immunity, leading to systemic immune improvement, which may contribute to its therapeutic effect in allergic asthma.

Keywords: Transdermal Topical Application; Immune Barrier; Asthma; Treating Winter Disease In Summer.

\section{Introduction}

Asthma, a chronic inflammatory disorder of the respiratory tract, is becoming a primary health burden throughout the world [1]. It affects estimated 300 million people worldwide and is concerned with growing incidence, prevalence and mortality [2]. This disease is characterized by airway inflammation, airway hyperresponsiveness, mucous edema, mucus hypersecretion and airway remodeling that leads to episodes of wheezing, coughing and shortness of breath [3, 4]. Currently, inhaling corticosteroids are the firstline therapy for control of airway inflammation in patients with asthma. However, these drugs will cause some side effects such as dysphonia, thrush, adrenal suppression and osteoporosis, when their dosage are to be increased [5]. Moreover, in spite of inhaled corticosteroids treatment $[3,6]$, nearly half asthmatics fail to benefit from one or more of these drugs [7]. Therefore, it is urgently required to develop new treatments for these chronic disease.

Recently, it was reported that IL-10 may play important roles in the pathogenesis of asthma, which is a vital cytokine secreted by helper T lymphocytes [8]. Furthermore, IL-10 promotes the development of a Th2 response to antigens and being indispensable for skin infiltration of eosinophils [9]. IFN- $\gamma$ is a typical Th1 cytokine, which is involved in plenty of immune regulatory

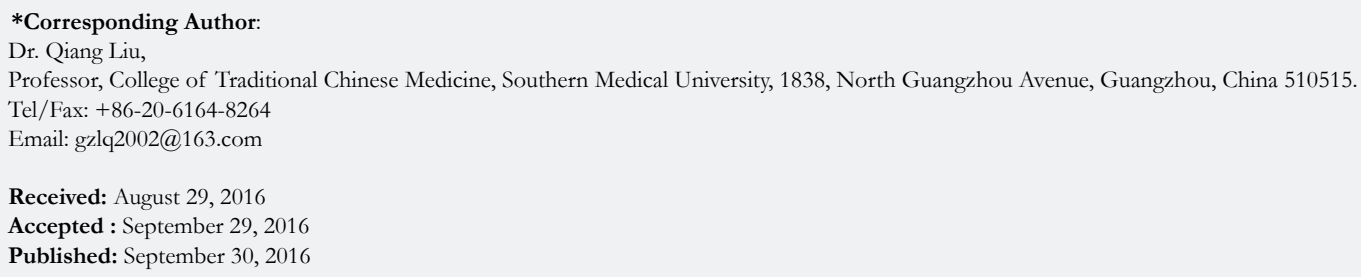

Citation: Shijian Xiang, Huoji Chen, Ting Zuo, Junhui Hong, Qiang Liua, et al., (2016) Effect of 'Treating Winter Disease in Summer' Prescription on Skin Immune Barrier and Systemic Immunity in a Guinea Pig Model of Asthma. Int J Clin Dermatol Res. 4(2), 81-86. doi: http://dx.doi.org/10.19070/2332-2977-1600022

Copyright: Liua $\mathbf{Q}^{\circ}$ 2016. This is an open-access article distributed under the terms of the Creative Commons Attribution License, which permits unrestricted use, distribution and reproduction in any medium, provided the original author and source are credited. 
mechanisms including up-regulation of pathogen recognition, antigen processing and presentation, otherwise, inhibition of cellular proliferation and activation of microbicidal effector functions, immunomodulation [10]. Th1/Th2 imbalance is the nuclear immune mechanism of allergic diseases, therefore, IFN- $\gamma$ may be a critical cytokine of allergic diseases. IFNs are evolutionarily preserved innate cytokines with pleiotropic function. IFN- $\alpha$, which belongs to the Type I IFN family, can promote the IL-10 expression, results in an anti-inflammatory state [11]. Previous works above show the importance of studying the expression of IL-10, IFN $-\alpha$ and IFN- $\gamma$ in the pathogenesis of asthma.

To treat some winter diseases in summer with transdermal application is widely used as an alternative medicine method for treating and clinical prevention of asthma. The long history and current popularity of the application of traditional Chinese Medicine (TCM) for asthma implies its potential advantages in the efficacy, including alleviating asthma symptoms, reducing the frequency of exacerbation, and improving the quality of life in asthma patients [12]. Drugs used in transdermal application for "treatment of winter disease in summer" are derived from a prescription in "Zhang's Treatise on General Medicine" written by Zhang Lu, an excellent doctor in the Qing Dynasty [13]. The prescription consists of white mustard (Sinapis alba L.), asarum (Asarum sieboldii Miq.), kansui root (Euphorbia kansui T. N.Liou ex T.P.Wang), rhizoma corydalis (Corydalis yanhusuo W.T.Wang), ephedra (Ephedra sinica Stapf) and scutellaria (Scutellaria baicalensis Georgi). That therapy is widely applied in China for its effectiveness and fewer side effects. According to statistics, asthma affects approximately 300 million people worldwide [7] and there are several hundred thousand asthma patients in China receive treatment of the prescription application in the summer of 2007 [12]. So it may be an alternative path for asthma treatment in abundant traditional medicine in the world. Although many studies have been done on the efficacy research of 'Treating Winter Disease in Summer' prescription, the underlying mechanism for the medication remains unclear. During the treatment of this prescription, because of its percutaneous drug delivery way, we suppose that the prescription may regulate systemic immune by means of affecting skin immune, ultimately, to achieve the effect of the treatment of asthma. On the other hand, skin, the largest organ of the human body [14], is not only being a physical barrier, but also regarded as a organ possessing immune function and related to the systemic immune of human body. Similar to intestinal mucosa and lung barriers, the skin exploits the immune supervision polyfunctionality of a well-coordinated system of epidermal and immune competent cells, such as Langerhans cells, leukomonocyte, etc [15]. In general, the cutaneous immune system is divided into an innate and an adaptive part. The skin is not only required to perform various missions in order to maintain homeostasis crucial for health, but also served as an immune protective organ that actively safeguards deeper body tissues [16]. There are many components in the skin that are mediating immune response, such as cytokines, immunoglobulins and neuropeptide, etc. These components make up the immune barrier in topical skin. It is important for skin to ensure that initiative safeguard and regulatory mechanisms are functioning well to keep the homeostatic balance between appropriate beneficial immune response and unnecessary immune responses [16]. The skin also secretes cytokines involved in multifarious immune responses, which is to regulate immune response in skin immune barrier accordingly to keep the balance of the systemic immune function. Appropriate immune function of the skin is crucial, for the skin exerts a protective function as a physical barrier and also a tool of initial recognition of extraneous substances as to determine about the induction or inhibition and the quality of immune responses [16].

Because of the unclear underlying mechanism of the medication for asthma, this area is still a hot spot of study. In this study, we investigate the skin immune barrier and systemic immunity by testing immune-related proteins with molecular biology methods, in order to obtain the theoretical foundation for the mechanism of treatment of asthma.

\section{Materials and Methods}

\section{Materials}

The preparation of Transdermal Drug delivery (TDD) patches: white mustard (Sinapis alba L.), asarum (Asarum sieboldii Miq.), kansui root (Euphorbia kansui T. N.Liou ex T.P.Wang), rhizoma corydalis (Corydalis yanbusuo W. T. Wang), ephedra (Ephedra sinica Stapf) and scutellaria (Scutellaria baicalensis Georgi) were shattered into powder according to the proportion of weight (2:2:1:2:1:1). Then mix $10 \mathrm{~mL}$ Succus Zingiberis to $10 \mathrm{~g}$ powder above to make patches. All of the Chinese herbal medicine above was purchased from Zhixin Medicine Health CO., LTD (Guangzhou, China). The plant was authenticated by Professor Chuanming Liu of College of Chinese Traditional Medicine, Southern Medical University, Guangzhou, China. Baicalin, Asarinin, ephedrine hydrochloride, tetrahydropalmatine, sinapine thiocyanate were obtained from The National Institute for the Control of Pharmaceutical and Biological Products (China). Methanol and acetonitrile were purchased from Oceanpak (Sweden). Hematoxylin and eosin stain, Ovalbumin (OVA, grade V, purity $\geq 98 \%$ ) were purchased from Sigma-Aldrich (St Louis, MO, USA). IL-10 antibody, IFN- $\alpha$ antibody and IFN- $\gamma$ antibody were purchased from Antibodiesonline (Atlanta, USA). $\beta$-actin and Secondary antibody were purchased from Boster (China). Polyvinylidene fluoride (PVDF) membrane was obtained from Millipore Corporation (Bedford, MA, USA). ECL (Western Blotting Substrate) was from Pierce Corporation (Pierce, USA).

\section{Animals and disposition}

Guinea-pig (male and female, 250-300g) were obtained from the Experimental Animal Center in Guangzhou University of Chinese Medicine (Guangzhou, China). Animals were kept under the experimental conditions (temperature: $20 \pm 2{ }^{\circ} \mathrm{C}$, humidity: $60 \pm$ $5 \%$ and $12 \mathrm{~h}$ dark/light cycle) for one week. All work performed with animals was in compliance with the Use and Care of Laboratory Animals and ethical rules of the Experimental Animal Center in Guangzhou University of Chinese Medicine, and it has been approved (Approval Number: SYXK GD 2014-0023). All surgery was performed under anesthesia by $3 \%$ pentobarbital sodium, and all efforts were made to minimize suffering of animals.

After one-week adaptation, guinea-pigs were randomly divided into blank control group, OVA sensitization model group and TDD patch therapeutic group each containing ten animals. Animals of OVA group and TDD patch therapeutic group were sensitized with intraperitoneal injection of $100 \mathrm{mg}$ OVA(Grade $\mathrm{V}$ Sigma-Aldrich) plus $1 \mathrm{mg}$ of $\mathrm{Al}(\mathrm{OH})_{3}$ as an adjuvant in $1 \mathrm{~mL}$ saline on Days 0 and 7. On Days 15-28, the OVA sensitized guinea- 
pigs were challenged by inhaling an aerosol containing 1\% OVA for $10 \mathrm{~min}$. Blank controlled group animals received $0.9 \%$ saline $[1,19]$. On days 29-42, guinea-pigs of TDD patch group received transdermal administration of TDD patches $(1 \mathrm{~g} / 100 \mathrm{~g}$, once daily) on the back affected areas. On day 43, all of guinea-pigs were anesthetized by intraperitoneal injection of $10 \%$ chloral hydrate anesthesia in saline after $24 \mathrm{~h}$ of the last treatment. Under anesthesia, all the lung tissues and skin tissues were obtained from the guinea-pigs, and then were used for the histological analyses, or stored at $-80^{\circ} \mathrm{C}$ for protein measurement.

\section{HPLC analysis}

Powder of 'Treating Winter Disease in Summer' prescription $(9 \mathrm{~g})$ was ultrasound-extracted with methanol $(100 \mathrm{~mL})$ for $30 \mathrm{~min}$. Moreover, Each kind of powder $(1 \mathrm{mg})$ of baicalin, asarinin, ephedrine hydrochloride, tetrahydropalmatine, sinapine thiocyanate were all dissolved in methanol $(10 \mathrm{~mL})$ as mixed reference substances. Lastly, the samples above were ready for HPLC analysis after being filtered by means of a filter $0.45 \mu \mathrm{m}$. Samples were analyzed by high-performance liquid chromatography (HPLC), equipped with a quaternary pump (both model series 1260; Agilent Technologies Inc., Wilmington, DE, USA), on-line vacuum degasser, auto-sampler, column temperature controller, diode array, and DAD detector, along with an analytical workstation. For the analysis, a HC-C18 column $(4.6 \mathrm{~nm} \times 250 \mathrm{~mm}, 5 \mu \mathrm{m}$, Agilent Technologies, USA) was used. Injection volume was $10 \mu \mathrm{L}$. Mixture of acetonitrile (A) and distilled water containing $0.1 \%$ phosphoric acid (B) was as eluent. The gradient elution was as follows: 0-16min, 5-24\% A, 16-30min, 24-36\% A, 30-60min, 36$69 \% \mathrm{~A}, 60-80 \mathrm{~min}, 69-90 \% \mathrm{~A}, 80-85 \mathrm{~min}, 90-95 \% \mathrm{~A}$. The flow rate is $1.0 \mathrm{~mL} / \mathrm{min}$. Column temperature was held at $25^{\circ} \mathrm{C}$. Detection was performed by a DAD detector and a wavelength of $250 \mathrm{~nm}$.

\section{Histology}

Lung and skin tissues were fixed in $4 \%(\mathrm{w} / \mathrm{v})$ paraformaldehyde, dehydrated and embedded in paraffin. Paraffin blocks were sectioned into $4 \mu \mathrm{m}$-thickness slices with a RM2245 microtome (Leica Microsystems, Germany). The slices of lung tissues were stained with hematoxylin-eosin (H\&E) according to standard protocols [18] and finally visualized under an optical microscope (LV100D, Nikon, Japan) with $100 \times$ zoom objectives. Images analysis of pathological sections included the study of the following markers relevant to asthma: airway condition, inflammatory cells, lymphocytes and eosinophilic granulocytes.

\section{Immunohistochemistry}

To study the skin immune barrier behavior during dermal administration, IL-10, IFN- $\alpha$ and IFN- $\gamma$ were detected by immunohistochemistry. Paraffin sections $(4 \mu \mathrm{m})$ were antigen repaired with citrate buffer acid buffer and incubated with $3 \%$ hydrogen peroxide for $10 \mathrm{~min}$ in order to block endogenous peroxidase follow by blocked in 5\% bovine serum albumin for $30 \mathrm{~min}$. Sections were incubated with the primary antibody for $12 \mathrm{~h}$ at $4 \mu \mathrm{m}$. After washing with PBS three times in $5 \mathrm{~min}$, the sections were incubated with HRP-labeled secondary antibody for $30 \mathrm{~min}$ and washed with PBS, followed by adding DAB solution for coloration and finally counter stained with hematoxylin. The sections were imaged with an optical microscope (LV100D, Nikon, Japan). Brown particles appeared to represent positive staining. The distribution and integrated optical density (IOD) of positive expression were measured with Image-Pro plus 6.0 image analysis software and then were statistically analyzed.

\section{Western blotting analysis}

The indexes were quantified using western blot method. Skin homogenates were prepared from control as well as OVA-sensitized guinea-pigs untreated or treated with TDD patches according to the instruction of membrane protein extraction kit and then using BCA method to determinate the concentration of proteins. Samples containing equal amounts of protein were added to $2 \mathrm{x}$ sample buffer, and $12 \%$ SDS-PAGE, transferred to PVDF membrane by electroblotting at $100 \mathrm{~V}$ for $2 \mathrm{~h}$. After being transferred, the membrane was incubated with primary antibodies overnight following treatment with $5 \%$ nonfat milk in TBS for $2 \mathrm{~h}$ and washed three times with PBS for 5 min each time. The membrane was then incubated with secondary antibody at a 1:2000 dilution for 1 h. The image development was detected with a ECL system. Automatic image analytic system was then used to measured the average grey value. The primary antibodies in this experiment were IL-10 antibody (1:200, Antibodies-online, Atlanta, USA), IFN- $\alpha$ antibody (1:200, Antibodies-online, Atlanta, USA) and IFN- $\gamma$ antibody. (1:200, Antibodies-online, Atlanta, USA)

\section{Measurement of serum IL-10, IFN- $\alpha$ and IFN- $\gamma$ by ELISA}

Serum was collected from all guinea-pigs. Enzyme-linked immunosorbent assay (ELISA) was used to determine the concentrations of IL-10, IFN- $\alpha$ and IFN- $\gamma$ in serum according to the manufacturer's instructions. ELISA kits were obtained from Antibodies-online (Atlanta, USA).

\section{Statistical analysis}

All values are expressed as means $\pm \mathrm{SD}$. Statistical significance among experimental groups was evaluated by one-way ANOVA or Student's t-test. A difference of $\mathrm{P}<0.05$ was considered statistically significant, and $\mathrm{P}<0.01$ was considered highly significant.

\section{Results}

\section{HPLC analysis}

The components identification of 'Treating Winter Disease in Summer' prescription was determined using HPLC. Typical HPLC chromatograms are shown in Figure 1. According to the study previously, sinapine thiocyanate is the main component of white mustard; asarinin is the main component of asarum; tetrahydropalmatine is the main component of rhizoma corydalis; ephedrine hydrochloride is the main component of ephedra and baicalin is the main component of scutellaria [18]. As is illustrated in B of Figure.1, there can be confirmed that the sample of Treating Winter Disease in Summer' prescription contains sinapine thiocyanate, asarinin, tetrahydropalmatine, ephedrine hydrochloride and baicalin, comparing with A. It turned out that 'Treating Winter Disease in Summer' prescription is authentically including white mustard, asarum, kansui root, rhizoma corydalis, ephedra and scutellaria. 
Figure 1. Chromatogram of Mixed Reference Substances (A), and Prescription Fingerprint (B).

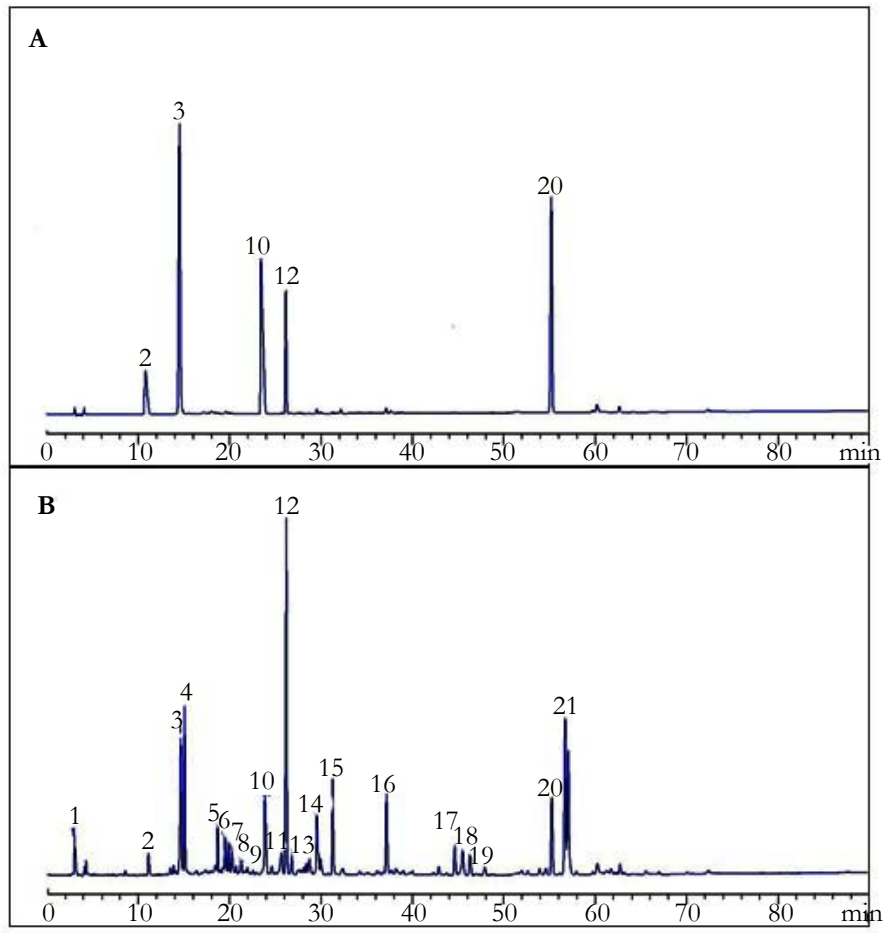

2-Ephedrine hydrochloride 3-Sinapine thiocyanate 10-Tetrahydropalmatine 12-Baicalin 20-Asarinin

\section{Hematoxylin and eosin $(\mathrm{H} \& \mathrm{E})$ staining}

Histological analysis performed on lung sections derived from blank control group, OVA sensitization model group as well as TDD-patch-treated guinea-pigs were stained with Hematoxylin and eosin $(\mathrm{H} \& \mathrm{E})$. The results are presented in Figure 2. Histological analyses of stained lung sections showed that high numbers of inflammatory cells, including lymphocytes and eosinophilic granulocytes in OVA sensitization model group compared to the blank control group, indicating that guinea-pig asthma model was successfully established. Airway inflammation cells were reduced in TDD patch therapeutic group compared with OVA sensitization model group.

\section{Immunohistochemistry}

Immunohistochemistry analysis was performed on the skin tissue derived from guinea-pigs of blank control group, OVA sensitization model group as well as in TDD patch therapeutic group. Histological analyses of stained skin sections are as follows: positive expression of membrane and showing stained brown particles in the cytoplasm, the deeper the color, the stronger expression, no appearance of brown particles is negative. As is illustrated in Figure 2, the expression of IL-10, IFN- $\alpha$ and IFN- $\gamma$ cytokines in skin was down-regulated induced by OVA-sensitization compared with the expression levels of cytokines detected in control guineapigs. However, following TDD patches treatment, data analyses revealed a significant increase in IL-10, IFN- $\alpha$ and IFN- $\gamma$ levels relative to the expression in OVA-sensitized guinea-pigs.

\section{Western blotting}

Western blotting analysis of samples was conducted to directly detect the IL-10, IFN- $\alpha$ and IFN- $\gamma$ contents in the skin. As is shown in Figure 3, an obvious increased level of IL-10, IFN- $\alpha$ and IFN- $\gamma$ were observed from the results of western blot after the administration of TDD patches, which exhibited a same trend of variation with the result of immunohistochemistry.

\section{Serum levels of IL-10, IFN- $\alpha$ and IFN- $\gamma$}

For specifically studying more about the effects to the immune responses in a guinea pig allergic asthma model, the secretion of various cytokines in serum was measured by ELISA. As shown in Figure 4, the levels of IL-10, IFN- $\alpha$ and IFN- $\gamma$ were entirely different among the three experimental groups. Significant decrease of IL-10, IFN- $\alpha$ and IFN- $\gamma$ level in OVA sensitization model group $(\mathrm{P}<0.05)$ was observed, which compared with the blank group. However, compared with OVA sensitization model group, there is significant increase of IL-10, IFN- $\alpha$ and IFN- $\gamma$ level in TDD patch therapeutic group $(\mathrm{P}<0.05)$.

\section{Discussion}

Allergic asthma is the result of an allergen exposure, and is representatively characterized by an immediate or early-phase response, such as abrupt onset of bronchoconstriction, airway inflammatory and hyperresponsiveness [19]. The guinea-pig asthma model has proven valuable for study on the pathogenesis and therapeutic mechanism of asthma. In this study, we investigated the effect of transdermal topical application of 'Treating Winter Disease in Summer' prescription on skin tissue and serum in an allergic model of asthma. According to the result of Hematoxylin and eosin (H\&E) staining, the lung sections of guinea-pigs in OVA sensitization model group showed hyperplasia and hypertrophy in goblet cells, bronchoconstriction as well as high numbers of inflammatory cells, including lymphocytes and eosinophilic granulocytes. It is indicated that the guinea-pig asthma model was successfully established. After the treatment of TDD patches, airway 
Figure 2. H\&E staining of guinea-pig lung sections and immunohistochemical staining of guinea-pig skin sections including IL-10, IFN- $\alpha$, IFN- $\gamma$. Cells which are positive have been specified by arrows. Magnification: 100 times.

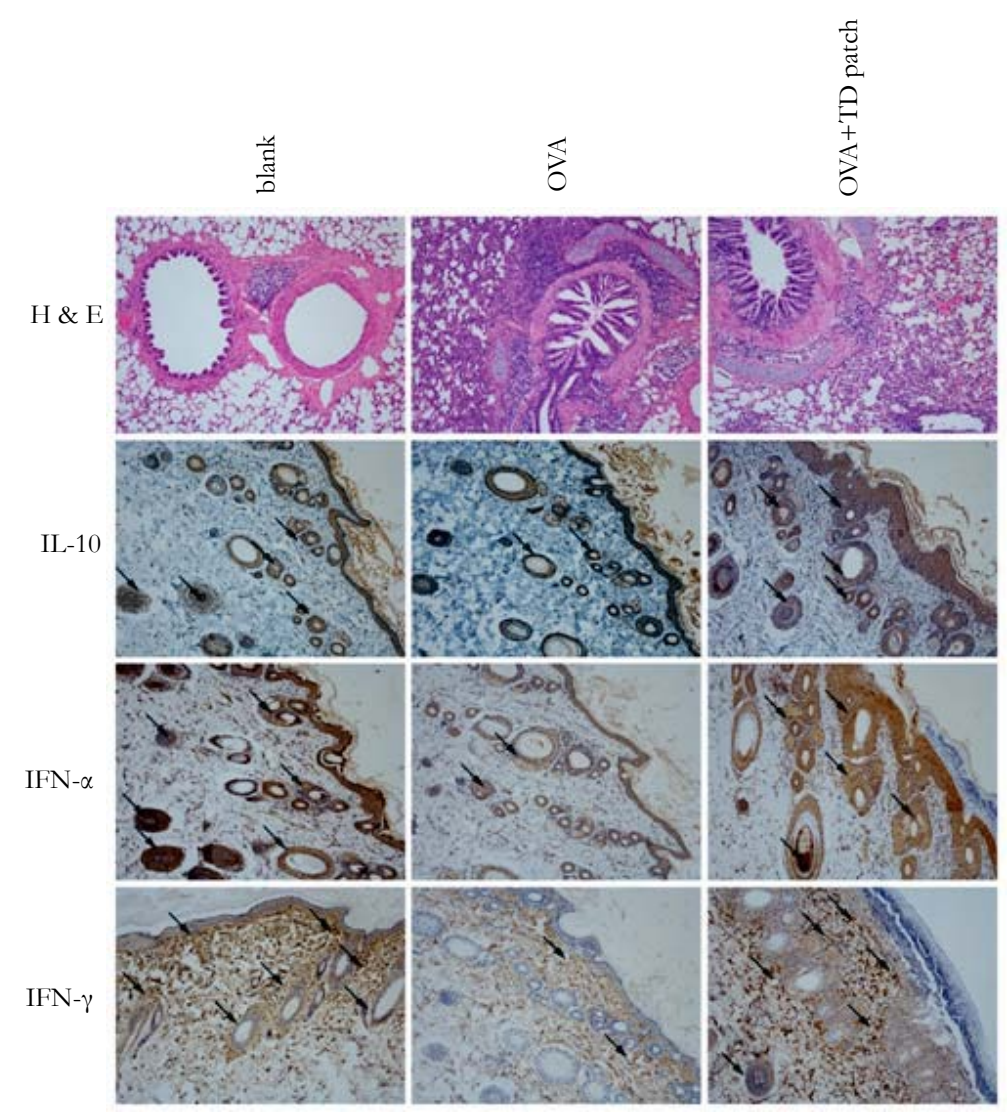

Figure 3. The expression of IL-10, IFN- $\alpha$ and IFN- $\gamma$ by Western blotting in skin tissue. (A)Typical Western blotting and (B) subsequent quantitative analysis of IL-10, IFN- $\alpha$ and IFN- $\gamma$. All data are shown as means \pm SD $(n=6, * P<0.05)$.
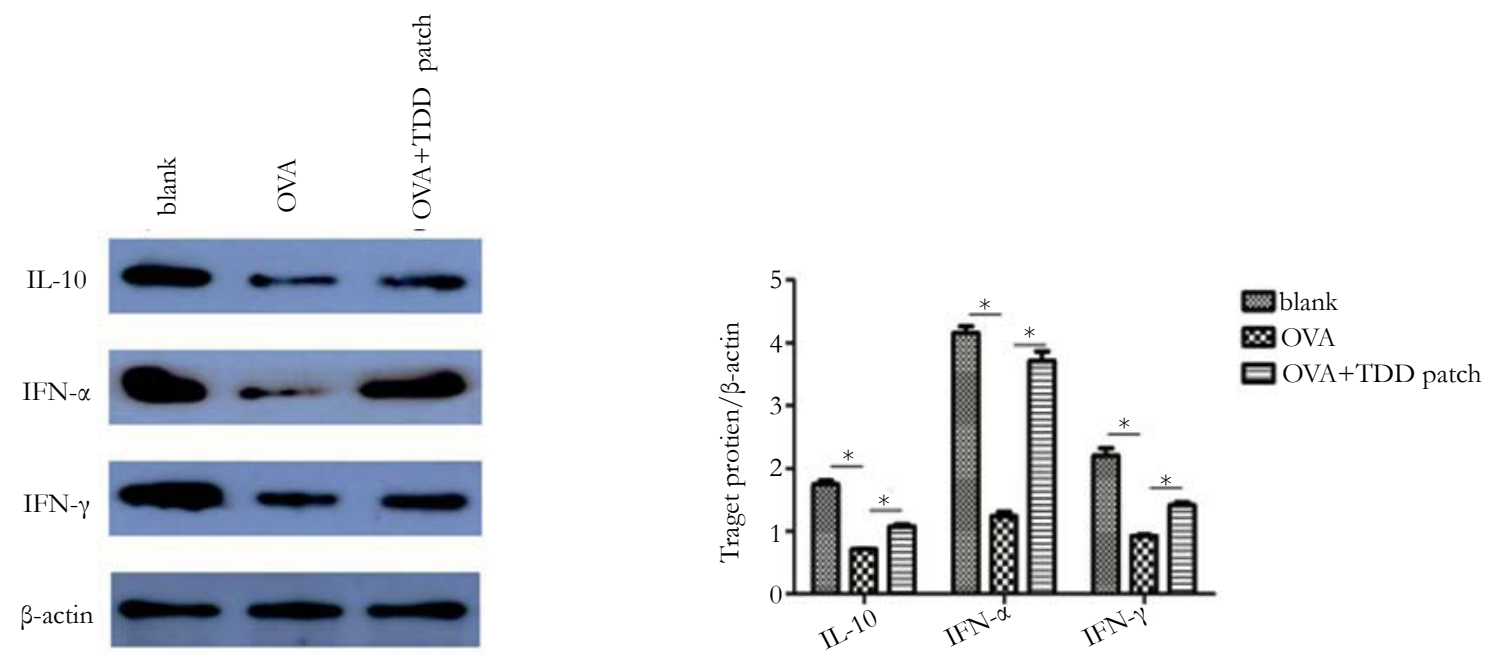

hyperresponsiveness and inflammation of guinea-pigs was significantly alleviated. Hence the TDD patches has favorable curative effect in asthma therapy.

As the largest organ of the human body, skin is not only being a physical barrier, but also regarded as a organ possessing immune function and related to the systemic immune of human body. It serves as a wide range of perception, regulatory and mechanical functions [22]. We propose that cytokine-mediated immunity in system as well as topical skin may play a crucial role in the modulation of airway inflammation. It has previously been reported that IFN- $\alpha$ can regulate the balance between pro-inflammatory and anti-inflammatory cytokines and increase the secretion of IL-
10 [11]. And IL-10 is an efficient anti-inflammatory cytokine with apparent effects on both innate and adaptive system, which can be generated by multiple immune cell types. Furthermore, it plays a vital role in relieving immune-mediated inflammation in allergy and immunity, such as allergic asthma [21] and was reported to redirect pathologic allergic responses by a broad range of suppressive mechanisms [22]. Therefore, the change of IFN- $\alpha$ and IL-10 level in guinea-pigs is particularly crucial. On the basis of our current data, it is demonstrated that both of the IL-10 and IFN- $\alpha$ expression were significantly increased in skin tissue as well as serum from animals receiving TDD patch therapy. Thus, we can speculate that TDD patch treatments may increase the IL-10 by up-regulating the level of IFN- $\alpha$. Previous studies revealed that 
Figure 4. The Levels of IL-10, IFN- $\alpha$ and IFN- $\gamma$ by Enzyme-linked Immunosorbent Assay in Serum of Guinea-pigs. All Data are Shown as means \pm SD $(n=10, * P<0.05)$.

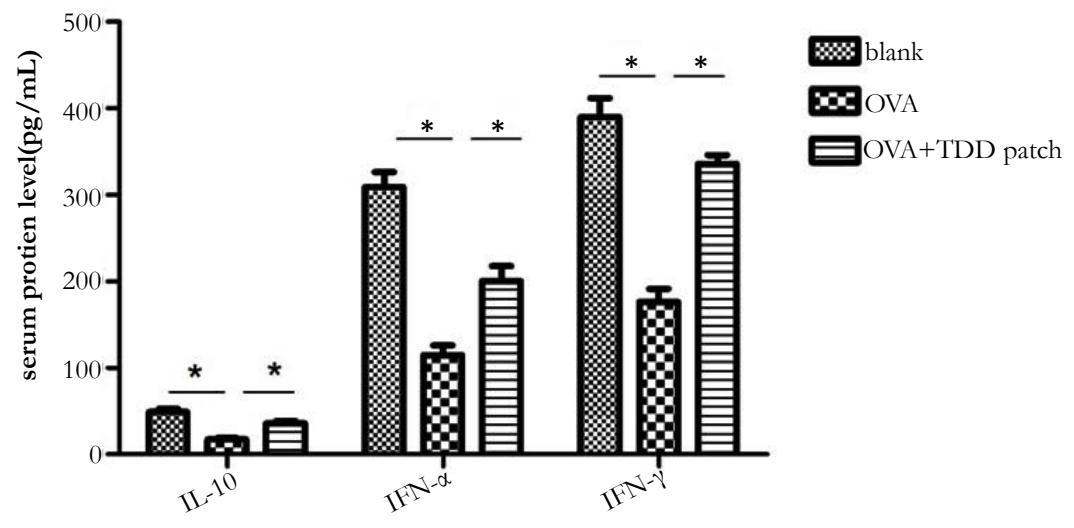

IFN- $\gamma$ is a Th1 cytokine and is known to have anti-allergic properties [23], it may act to resist a Th2 response and was involved in suppressing late-phase allergic airway responses. Our study shows that TDD patch treatments increased the expression levels of IFN- $\gamma$ in skin tissue and serum of asthma guinea-pigs as well.

In summary, the study above fully illustrates that all the expression of IL-10, IFN- $\alpha$ and IFN- $\gamma$ in skin and serum of asthma guinea-pigs were significantly increased, and airway inflammation condition was reduced by the treatment of TDD patches. The trend of the expression of these three immune factors is highly consistency between in skin and in serum. It is demonstrated that transdermal delivery of 'Treating Winter Disease in Summer' prescription may improve skin immunity, leading to systemic immune adjustment, which may contribute to its therapeutic effect in modulating inflammation and brochoconstriction in asthma. Nevertheless, further studies are required in order to elucidate the immunity protective mechanism of 'Treating Winter Disease in Summer' prescription treated in summer to asthma through transdermal drug delivery.

\section{Acknowledgments}

We gratefully thank the Natural Science Foundation of China (No.81373975) for financial support.

\section{References}

[1]. Sagar S, Akbarshahi H, Uller L (2015) Translational value of animal models of asthma: Challenges and promises. Eur J Pharmacol. 759: 272-277.

[2]. Bahadori K, Doyle-Waters MM, Marra C, Lynd L, Alasaly K, et al., (2009) Economic burden of asthma: a systematic review. BMC Pulm Med. 9: 1-16.

[3]. Yonetomi Y, Sekioka T, Kadode M, Kitamine T, Kamiya A, et al., (2015) Effects of ONO-6950, a novel dual cysteinyl leukotriene 1 and 2 receptors antagonist, in a guinea pig model of asthma. Eur J Pharmacol. 765: 242-248.

[4]. Ablimit A, Hasan B, Lu W, Qin W, Wushouer Q, et al., (2013) Changes in water channel aquaporin 1 and aquaporin 5 in the small airways and the alveoli in a rat asthma model. Micron. 45: 68-73.

[5]. FitzGerald JM, Shahidi N (2010) Achieving asthma control in patients with moderate disease. J Allergy Clin Immunol. 125(2): 307-311.

[6]. Williamson PA, Short PM, Vaidyanathan S, Lipworth BJ (2013) Inhaled and systemic corticosteroid response in severe asthma assessed by alveolar nitric oxide: a randomized crossover pilot study of add-on therapy. $\mathrm{Br} \mathrm{J}$ Clin Pharmacol. 75(1): 93-102.

[7]. Bosnjak B, Stelzmueller B, Erb KJ, Epstein MM (2011) Treatment of allergic asthma: modulation of Th2 cells and their responses. Respir Res. 12: 114.

[8]. Zhang YL, Luan B, Wang XF, Qiao JY, Song L, et al., (2013) Peripheral blood MDSCs, IL-10 and IL-12 in children with asthma and their importance in asthma development. PLoS One. 8 (5): e63775.

[9]. Lee J, Noh G, Lee S, Youn Y, Rhim J (2012) Atopic dermatitis and cytokines: recent patents in immunoregulatory and therapeutic implications of cytokines in atopic dermatitis--part I: cytokines in atopic dermatitis. Recent Pat Inflamm Allergy Drug Discov. 6(3): 222-247.

[10]. Singh N, Bhattacharyya D (2014) Collagenases in an ether extract of bacterial metabolites used as an immunostimulator induces TNF-alpha and IFNgamma. Int Immunopharmacol. 23 (1): 211-221.

[11]. Touzot M, Cacoub P, Bodaghi B, Soumelis V, Saadoun D (2015) IFN-alpha induces IL-10 production and tilt the balance between Th1 and Th17 in Behcet disease. Autoimmun Rev. 14(5): 370-375.

[12]. Wen BL, Liu BY, Jin P, Wang XF, Xiang XX, et al., (2012) Clinical research of acupoint application for "treatment of winter disease in summer" used to prevent and treat bronchial asthma inchildren. J Tradit Chin Med. 32(1): 31-39.

[13]. Zhang L (1995) Zhang's Treatise on General Medicine in the Qing Dynasty. Chinese TCM Press.

[14]. Skabytska Y, Kaesler S, Volz T, Biedermann T (2016) The role of innate immune signaling in the pathogenesis of atopic dermatitis and consequences for treatments. Semin Immunopathol. 38(1): 29-43.

[15]. Wachholz S, Eßlinger M, Plümper J, Manitz MP, Juckel G, et al., (2016) Microglia activation is associated with IFN-alpha induced depressive-like behavior. Brain Behav Immun. 55: 105-113.

[16]. Di Meglio P, Perera GK, Nestle FO (2011) The multitasking organ: recent insights into skin immune function. Immunity. 35 (6): 857-869.

[17]. Morin C, Fortin S, Cantin AM, Rousseau É (2013) MAG-EPA resolves lung inflammation in an allergic model of asthma. Clin Exp Allergy. 43 (9): 10711082.

[18]. National Pharmacopoeia Committee (2015) Pharmacopoeia of People's Republic of China. Chemical Industry Press. Beijing ,pp. 1: 88,139-140,160161,301-302,320-321.

[19]. Murad HA, Hasanin AH (2014) The anti-inflammatory effects of 1, 1 dimethyl-4-phenylpiperazinium (DMPP) compared to dexamethasone in a guinea pig model of ovalbumin induced asthma. Eur Rev Med Pharmacol Sci. 18(15): 2228-2236.

[20]. Guo R, Xu S, Ma L, Huang A, Gao C (2011) The healing of full-thickness burns treated by using plasmid DNA encoding VEGF-165 activated collagen-chitosan dermal equivalents. Biomaterials 32 (4): 1019-1031.

[21]. Chaudhry A, Samstein RM, Treuting P, Liang Y, Pils MC, et al., (2011) Interleukin-10 signaling in regulatory $\mathrm{T}$ cells is required for suppression of Th17 cell-mediated inflammation. Immunity. 34(4): 566-578.

[22]. Ji P, Hu H, Yang X, Wei X, Zhu C, et al., (2015) AcCystatin, an immunoregulatory molecule from Angiostrongylus cantonensis, ameliorates the asthmatic response in an aluminium hydroxide/ovalbumin-induced rat model of asthma. Parasitol Res. 114(2): 613-624.

[23]. Lee JH, Noh G (2013) Polydesensitisation with reducing elevated serum total IgE by IFN-gamma therapy in atopic dermatitis: IFN-gamma and polydesensitisation (PDS). Cytokine. 64 (1): 395-403. 